\title{
La actividad informativa en los medios digitales: ¿sobrevivirán los periódicos impresos sin publicidad?
}

\author{
Juan Carlos Marcos ReCiO \\ Universidad Complutense de Madrid \\ jmarcos@ucm.es \\ Pedro García-Alonso \\ Universidad Complutense de Madrid \\ pedro.garciaalonso@ccinf.ucm.es \\ David PARRA VAlCARCE \\ Universidad Complutense de Madrid \\ davidparra@ccinf.ucm.es
}

Recibido: 2 de diciembre de 2013

Aceptado: 5 de mayo de 2014

\section{Resumen}

La crisis ha acelerado el proceso de digitalización en la prensa impresa. Las TIC han transformado el sistema informativo, tanto económica, como productivamente. El descenso dramático en ingresos publicitarios hace tambalearse el vigente modelo de negocio de las empresas informativas. Necesitamos nuevas vías de financiación, que permitan subsistir a los medios. Existen nuevos modelos de sistemas para conseguir dar rentabilidad a la actividad informativa: desde el crowdfunding, hasta el e-commerce, apoyado en sus audiencias, y otros. Todo eso nos hace cuestionarnos el futuro de los medios informativos, tal y como han sido entendidos hasta ahora, en cuanto empresas.

Palabras clave: Publicidad, periódicos impresos, medios digitales, futuro de los medios, nuevo modelo de negocio.

\section{Information activity in digital media: will print newspapers survive without advertising?}

\begin{abstract}
This crisis has accelerated printed press' process to become online. ICT have transformed the information system both economically and productively. The dramatic decline in advertising revenues shakes the business model followed so far by media companies. We need new ways of financing, to allowe media survive. There are cases of new systems adopted for information activity to obtain profitability: from crowdfunding to e-commerce, supported in media audiences. All this makes us question the future of the media, in the way as they have been understood as information companies untill now. Keywords: Advertising, print media, digital media, future of media, new business model.

\section{Referencia normalizada}

MARCOS RECIO, Juan Carlos; GARCÍA-ALONSO, Pedro; y PARRA VALCARCE, David (2014): "La actividad informativa en los medios digitales: ¿Sobrevivirán los periódicos impresos sin publicidad?”. Estudios sobre el Mensaje Periodístico. Vol. 20, Núm. 1 (enero-junio), págs.: 177-194. Madrid, Servicio de Publicaciones de la Universidad Complutense.

1 Este artículo, del Grupo de Investigación y Análisis de Internet en el Periodismo de la Universidad Complutense de Madrid (GIAIP-UCM), forma parte del proyecto de investigación "Supervivencia del periodismo en la era postdigital", financiado por el Ministerio de Ciencia e Innovación, a través del Plan Nacional de I+D+i.
\end{abstract}


Sumario: 1. Futuro de los medios. 2. Cómo la tecnología transforma los medios hacia lo digital: algunas claves; 2.1. Desaparición de los medios impresos; 2.2. Transformación/adaptación del impreso a lo digital; 2.3. Fusiones entre distintos medios, antes competencia; 2.4. Nuevas alternativas, como una aplicación que resume las noticias; 2.5. Proyectos para cambiar las reglas del periodismo: crowdfunding; 2.6. Otros proyectos: crowdfunding inverso. 3. La actividad informativa como valor para las empresas editoras ante la amenaza digital. 4. ¿La inversión publicitaria salvará los medios impresos y/o los digitales?; 4.1. Google frente a todos y contra todos; 4.2. España, un incierto futuro de los medios impresos. 5. Conclusiones. 6 . Referencias bibliográficas.

"My best guess is that mobile will be the lead channel for delivery within three to four years. [...] Mobile already accounts for 25\% of FT.com's traffic and is said to be driving between $15 \%-20 \%$ of all new subscriptions, where iPad users lead the charge".

Rob Grimshaw, jefe de edición digital de Financial Times.

\section{Futuro de los medios}

Recientemente, algunos gurús de la comunicación y de las tecnologías han puesto fecha a la desaparición de la prensa. Desde hace más de una década, muchos investigadores auguran un futuro muy oscuro para los medios de comunicación, presagiando la desaparición de los mass media impresos. La situación actual, las tendencias en boga y el futuro más cercano parecen corroborarlo. Sin embargo, la trasformación y la adaptación son las constantes vitales de nuestro tiempo, también para los medios.

Aunque los periódicos continúan, han tenido que adaptarse a los nuevos modelos y usos de sus lectores, pues los nuevos jóvenes leen en Internet. Para atraer a esos jóvenes lectores, hace falta descubrir un nuevo modelo de negocio rentable y adecuado, como hacen ya hoy las grandes empresas líderes en las redes sociales con sus audiencias.

El objetivo de este texto es presentar cómo los periódicos se están adaptando a la nueva situación, evolucionando frente a otros soportes, manteniendo su incorporación de valor y analizando el mercado publicitario. Así veremos si la prensa es realmente viable hoy.

\section{Cómo la tecnología transforma los medios hacia lo digital: algunas claves}

Todo cambio requiere una adaptación. En el caso de los medios de comunicación, españoles, este cambio se ha debido a la tecnológica y a las exigencias de los lectores, más que por el esfuerzo de los editores. En cualquier caso, no les queda ya más remedio que ofrecer productos y servicios rentables. Una vía es asociarse, como han hecho los editores, mediante acuerdos y fusiones, como Kiosko y Más, entre $A B C$ y El País, u Orbyt de El Mundo, con otros medios. Pero la digitalización es imparable: "El 60\% de los editores coincide: los periódicos serán solo digitales en 2020" (Cruz, 2012a). No es un dato meramente especulativo, como ha venido siendo hasta ahora: la cifra es cada vez más real. Los datos proceden del estudio: "La Prensa, 16052020, D.E.P. ¿Hay vida tras la muerte?”, realizado por MPP Global Solutions, uno de los líderes en el mercado de soluciones digitales para periódicos, con un interés especial en temas de información digital.

Paul Johnson, su director ejecutivo, precisó en la presentación de dicho estudio que con las ventas de los periódicos en papel en declive, a la vista del gran aumento 
de ventas de teléfonos inteligentes y tabletas para que los usuarios accedan a las ediciones digitales de periódicos y revistas, los editores tienen que asegurarse abarcar la distribución de contenido digital en múltiples plataformas. "MPP Global Solutions, the leading provider of Payment Services and eCommerce Solutions to the Media and Entertainment Industries conducted the exploration entitled 'Print Publishing, 1605 2020, R.I.P. Is There Life after Death?' [...] 'the future of print publishing is limited"' (Editor\&Publisher, 2013)

El convulso mundo de los medios de comunicación no para de ensayar fórmulas para intentar salvar la versión impresa, la que generaba lectores fieles; pero les falla la publicidad. Asistimos a un cambio radical: ya no deciden los editores, sino que el poder decisorio está ahora en manos de los lectores: será la propia audiencia quien decida qué le interesa y cómo quiere recibir la información. El futuro más plausible apunta a una única información, servida mediante diferentes soportes y plataformas, para ahorrar en redacción y producción. Las empresas informativas disponen de varios caminos para lograr ese cambio, que mostramos a continuación.

\subsection{Desaparición de los medios impresos}

Es una postura un tanto radical, aunque no exclusiva. Por ejemplo, el diario económico alemán Financial Times Deutschland dejará de publicarse tras la reunión del Consejo de Vigilancia de su empresa editora, Gruner+Jahr, según informó el Frankfurter Allgemeine Zeitung (FAZ). En esta misma línea, la revista semanal estadounidense Newsweek publicó su último número el 31 de diciembre de 2012. Por tanto, el semanario es únicamente digital desde el 1 de enero de este año.

\subsection{Transformación/adaptación del impreso a lo digital}

Éste sería el caso de Le Monde: 'El periódico de papel tiene futuro siempre y cuando se transforme' ("Le journal papier a un avenir, à condition qu'il se transforme", Le Monde, 2012). 'Acelera sus aplicaciones en Internet', pues lleva trabajando dieciocho meses esa transformación. También está Times-Picayune que ya ha dejado de ser diario para convertirse en trisemanal. Su editora, Advance Publications, pretende así ahorrar costes y centrarse en el digital. Igualmente actúa así el británico The Guardian, que se está planteando eliminar la edición impresa, ya que actualmente acumula unas pérdidas anuales de 44 millones de libras.

\subsection{Fusiones entre distintos medios, antes competencia}

Así sucede a The Telegraph, que informó recientemente que los rumores acerca del futuro del Financial Times no cesan. Probablemente opte por crear una empresa conjunta entre Financial Times y Thomson Reuters. Este medio apuesta por lo digital, ofertándose en teléfonos y tabletas en 2016, según cálculos de Rob Grimshaw, jefe del diario digital, quien ha asegurado que el paso al móvil será 'un cambio de mayor magnitud que el de lo impreso al ordenador' (Dirigentes Digital, 2012).

\subsection{Nuevas alternativas, como una aplicación que resume las noticias}

Es el caso de un invento del joven británico de 17 años Nick d'Aloisio: una aplicación para iPhone que resume artículos de prensa en algunas líneas, dando una idea de las 
noticias en poco tiempo: 'Mi generación no consume la información como antes', dice d'Aloisio en su defensa: "I designed Summly because I felt that my generation wasn' consuming traditional news anymore', said Nick" (The Telegraph, 2012).

\subsection{Proyectos para cambiar las reglas del periodismo: crowdfunding}

Así sucede en Matter (www.readmatter.com): apuesta por un formato largo (contrario a la postura anterior) de contenidos digitales de ciencia y tecnología, para extenderse luego a otras secciones. Ofrece reportajes en profundidad, sobre historias importantes que no estén suficientemente contadas, valorando textos más largos que los que habitualmente se publican en Internet. Su precio -0,99 dólares- y su lema "Pocas historias pero tan completas que pudieran convertirse en una publicación individual" ("Each story as an individual publication, it's a small book. It isn't a magazine, it isn't a website, it's Matter", Journalism.co.uk, 2012)- lo dejan bastante claro. Este proyecto se ha financiado a través de crowdfunding y ya ha recaudado 140.000 dólares.

\subsection{Otros proyectos: crowdfunding inverso}

En octubre de 2012, el periodista neozelandés Keight Ng experimentó una nueva forma de negocio y de información: el crowdfunding inverso. En base a un error informático el periodista tuvo acceso a miles de archivos confidenciales desde un ordenador público, dentro de una oficia de empleo, según cuenta Marcus Hurst (Hurst, 2012). El periodista explicó el tiempo que había empleado en la investigación y a cambio pedía contribuciones voluntarias. En poco más de un día había conseguidos más de 2.500 euros, frente a los 440 euros que le habría pagado un periódico impreso por esa pieza.

Cuando se hacen especulaciones, los resultados son siempre erróneos, pero en algunos casos válidos. Lo son, al menos, en el estudio de News\&Tech: un lugar especializado en tecnología y periodismo digital, impreso y productos híbridos, como se definen en su sitio web, donde recientemente han publicado el informe " 20 trends for 2020 ". En él se apuntan algunas de las claves que conducirán al periodismo dentro de una década: "Websites are more intelligent, designed with elements designed to mesh with how the human brain interacts with them. [...] news that is you and you alone. Hyper-targeted information is continually displayed on your mobile device. [...] The printed newspaper survives, but not in every American city and not every day of the week. 8 Papers are offered in more shapes and sizes [...] Quality sells: The search for the truth still important" (News\&Tech, 2012).

Hay incluso quien ya propone, con argumentos tecnológicos, una fecha en la que irán desapareciendo los periódicos impresos. Así, Ross Dawson publicó a mediados de 2012 un texto en su blog: Newspaper Extinction Timeline, en base a un estudio de la red estratégica Future Exploration Network (futureexploration.net/future-of-media) en el que se señala que el primero en salir de esa línea sería EE.UU. en 2017, seguido por el Reino Unido e Islandia en 2019 y Canadá y Noruega en 2020. En el caso de España, se alargaría hasta 2024. 


\section{La actividad informativa como valor para las empresas editoras ante la ame- naza digital}

En apenas dos décadas la estructura informativa en los medios, propuesta por algunos autores -entre ellos Orihuela (2002) y Salaverría (2005)-, ha venido adaptándose a nuevas fórmulas impuestas desde la tecnología. Esa transformación ha sido muy dolorosa, intentado posponer el cambio, pero la mudanza no tiene retroceso. Por eso, ni la creación de redacciones únicas, ni las fórmulas de producción separadas, ni los productos especializados para lo digital y lo impreso, han dado hasta ahora resultado. Algunos editores pretenden obtener un rédito a la información digital, para compensar la sangría del medio impreso, cobrando por los contenidos.

Pero exigir pago ahuyenta a la audiencia, reduce lectores, limita visitas y aleja la publicidad. "Desde un punto de vista comercial, lo que está claro que no funciona es exigir al lector un complejo formulario de suscripción, al que añades la obligación de incluir todos los números de tu tarjeta de crédito. Rebajar las barreras, hacer las cosas más fáciles y cobrar en pequeñas cantidades es una herramienta muy poderosa. Es muy interesante el caso del New York Times, en el que tienes que pagar una vez que has consumido su información un número de veces, 20 por ejemplo" (Wales, 2013).

Uno de los expertos en contenidos digitales en medios de comunicación, James Breiner, considera que la actividad informativa dará valor a las empresas editoras cuando se "creen contenidos que se adapten a múltiples dispositivos" (Breiner, 2012), especialmente las tabletas, ya que más de un $20 \%$ de los adultos en Estados Unidos cuenta con una. Eso supone el doble del porcentaje con respecto a 2011, según datos de un estudio del Centro Pew (Breiner, 2013).

En términos muy similares se expresa Cabrera, cuando escribe: "Los avances en el proceso de convergencia tecnológica, empresarial, profesional, de las redacciones y de los contenidos, favorecen la distribución multiplataforma de la información por diversos canales y medios, lo que asegura un mayor consumo por parte de las audiencias. Sin embargo, la distribución multiplataforma precisa aún de un mayor desarrollo de aplicaciones tecnológicas basadas en la conectividad y compatibilidad 'que permita tener sinergias entre todos los medios' (Benito, 2009: 7), para lo cual resulta necesaria la colaboración entre las empresas de comunicación y de desarrollo informático" (Cabrera, 2010).

Durante siglos, la información se ha venido realizando de una única manera y siempre con la idea de dar una respuesta a estos interrogantes: qué, quién, cuándo, cómo, dónde y por qué. Apenas existía un cambio significativo entre los medios impresos y los analógicos. El periodismo digital empezó adaptando los contenidos de la versión impresa. Y fracasó. Luego ha experimentado otras fórmulas que tampoco han resuelto nada. Será preciso, entonces, empezar por el otro lado; es decir, crear contenidos para formatos digitales y luego adaptarlos a todos los medios, elaborando un tipo de información que sirva para las diferentes plataformas. Esa debe ser la clave. Un experto en tecnologías y en medios digitales como Sam Kirkland lo viene repitiendo con cierta frecuencia: "Hay que publicar una vez y hacer que se adapte a todas las plataformas" (Kirkland, 2012), es decir, al móvil, la tableta, el online y el impreso.

Esto supone un cambio total y profundo, lo que se conoce en Estados Unidos como 'Diseño responsivo', que en palabras de Breiner: "Permite a los medios evitar contratar 
legiones de programadores informáticos para crear una plataforma única y así mostrar el contenido en cada una de las decenas de teléfonos móviles, tabletas y otros dispositivos utilizados para consumir las noticias" (Breiner, 2012). Se trata de buscar un sistema que no obligue a contratar más personas en redacción, de tal modo que sólo con las que ya cuentan, sean suficientemente capaces de crear ese tipo de contenidos multifuncionales.

Además, hay que pensar que los usuarios ya manejan más de un dispositivo cada día: casi siempre un móvil y una tableta o un ordenador portátil. Por eso, ya se distinguen dos tipos de lectores, en función del lugar donde leen las informaciones, según datos de un estudio del Centro Pew, citados por Breiner: "Los lectores tienden a utilizar las tabletas y los medios impresos para una lectura más pausada (una experiencia de lectura más relajada o 'lean-back'), mientras que el uso del móvil y de los sitios web en general implica una lectura más intensa de las noticias (o 'lean-forward'). En ambos casos, la manera en que se elabora la noticia es la misma. En definitiva, se trata de crear una información válida para todos los soportes, pues reduce costes de redacción y de programación, tan necesarios de aminorar en estos tiempos de crisis, donde los ingresos publicitarios han descendido tanto" (Breiner, 2012).

Otra manera de sacar rendimiento a los contenidos, por parte de las empresas editoras, es la creación de un muro de pago. Por ejemplo, The Globe and Mail puso en marcha un paywall, es decir, un sistema que impide a los usuarios de Internet el acceso a contenido de páginas web, en especial, el de noticias, informaciones y publicaciones académicas sin una suscripción de pago. El sistema puede ser totalmente cerrado, o más flexible, como es el caso de este medio, donde se permite leer diez artículos al mes de forma gratuita y pagar el resto. Pero no es el único, y la tendencia crece: The Guardian, The New York Times, etc., están utilizando ya este sistema de forma parecida. En el caso de éste último, para datos del año 2012: "Las Suscripciones digitales generarán 91 millones de dólares, según Douglas Arthur. El paywall, por su estimación, representará el 12 por ciento de las ventas totales por abonados, que se complementarán con 768,3 millones de dólares este año. Eso supone 52,8 millones más que la publicidad" (Ruiz, 2013).

Otro medio que recientemente levantó un muro de pago es el británico The Telegraph. En este caso, la oferta va dirigida a sus lectores en el extranjero. No es, por tanto, una moda pasajera. En Estados Unidos se ha duplicado en el último año el número de periódicos que cobran por los contenidos informativos digitales. Esa tendencia será alcista en otros países que toman como modelo lo que sucede en USA. Este sistema añade beneficios de lector, pero recorta ingresos de publicidad, al rebajar la audiencia.

En Alemania la tendencia también se dirige a crear muros de pago para buscar rentabilidad en los medios. Uno de los grupos editoriales más poderosos de Europa, Axel Springer apuesta porque su diario estrella, Die Welt, disponga ya de un método de pago. Mathias Doepfner, CEO de la editorial, lo dejó claro: "100.000 usuarios de pago valen más que un millón de lectores que no pagan” (Carretero, 2012). Así pues, 2013 hará ver que otros grandes periódicos europeos se suman a esta nueva fórmula de pago. 


\section{4. ¿La inversión publicitaria salvará los medios impresos y/o los digitales?}

La publicidad disponible se mantiene, es la misma, pero hoy los canales informativos se multiplican: son más bocas a comer de un mismo plato. Los ingresos publicitarios que han huido a la Web desde la prensa impresa, no volverán. Algunos esperaban que las versiones online revirtieran ingresos sobre las impresas; pero eso fracasó. Los medios tradicionales desaparecerán en algún momento futuro... pero no tan inmediato: seguirán conviviendo las versiones impresa y digital, buscando ambas mutuo beneficio.

Aunque no hay soluciones perfectas, sí hay algunas útiles, con distintos resultados. En la prensa impresa, el descenso en el número de ventas se ha de compensar con más publicidad y, en el caso de los digitales, deben cobrar por algunos contenidos para obtener ingresos y completarlos con la publicidad, para poder así dar beneficios.

Ante el problema del descenso en la inversión publicitaria, muy pronunciando en los últimos años, en Estados Unidos, señala Jim Harris en un texto publicado en la versión canadiense de Huffington Post, 'What's Really Killing Print Journalism': “Los ingresos por publicidad en los periódicos norteamericanos han caído a su nivel más bajo en 60 años, disminuyendo en picado de 65.500 millones de dólares en 2000 a 19.000 millones en 2012. La suma con la publicidad on-line mejora el resultado un poco, pero aún así es peor que las cifras de 1953 (Gráfico 1)" (Harris, 2012). Estos datos parecen claros: la inversión en medios impresos disminuye a tal velocidad que no tardando se quedará sin publicidad y tendrán que buscar otra manera de conseguir recursos.

\section{Newspaper Ad Revenue}

(A djusted for Inflation 1950-2012 in 2012 Dollars, in sus Billions)

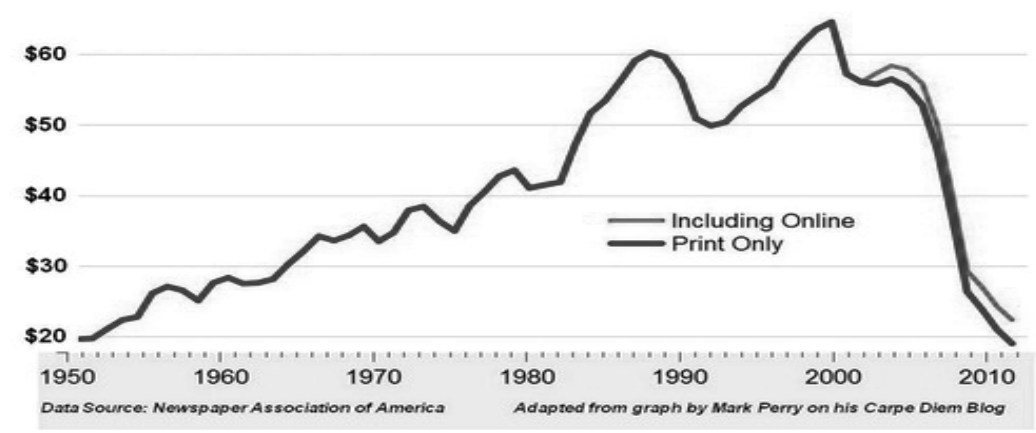

Fuente: Newspaper Association of America.

Quien sí cuenta con ingresos son los buscadores digitales. El futuro no es la publicidad, como señalan muchos expertos, sino las búsquedas que hagan los usuarios, es decir, el marketing a través de posicionamiento Search Engine Optimization (SEO) y también el gasto en motores de búsqueda y plataformas en las redes sociales. Estos datos lo avalan: "Entre el 35-42 por ciento del tráfico de búsqueda va al resultado número 1 en una búsqueda. Entre los tres mejores resultados, se logra el 58 por ciento 
del tráfico, mientras que el resultado del número 11 tiene menos del 1 por ciento del tráfico" ("Today this is critical as $35-42$ per cent of the search traffic goes to the \#1 result in a search, the top three results take 58 per cent of the traffic while the 11th result typically has less than 1 per cent of the traffic") (Harris, 2012).

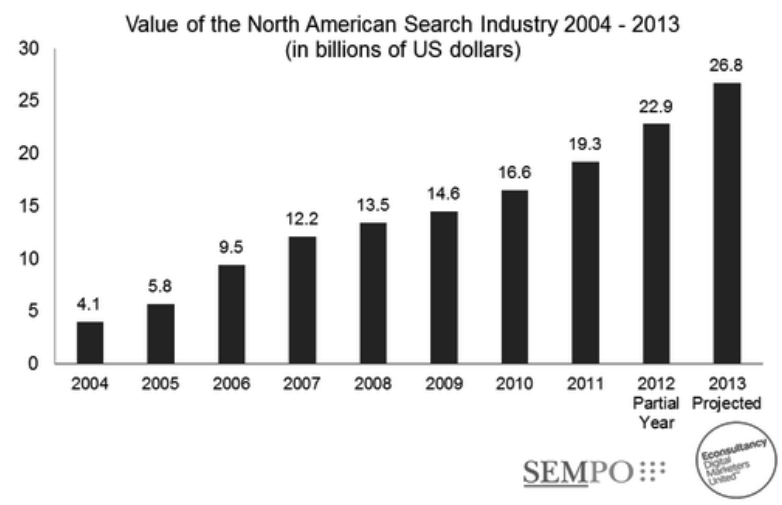

Fuente: SEMPO (Sample, 2012)

Otro problema es que los medios digitales crean productos con los que luego otras empresas se benefician. Son los llamados 'agregadores de noticias' o 'parásitos', arañas que rastrean la red y toman las mejores noticias para difundirlas luego en sus sitios web, beneficiándose a través de publicidad de esas informaciones. Además, esa publicidad ya no acude por tanto al medio digital que ha producido esa información. Este problema no es nuevo y hay editores que se han enfrentado a los todopoderosos Google, Facebook, etc. Por ejemplo, la Corporación de Medios de Rupert Murdoch solicitó a Google que dejara de sumar las noticias de The Times y de The Sunday Times.

Algunos autores sostienen que el periodismo impreso empezó a perder publicidad cuando en 2009 se crearon en Internet sitios con millones de anuncios del mercado, clasificados y gratuitos, en sitios tales como AutoTrader o Monster.com. Lo que hasta entonces había sido una sección importante para los medios impresos -Anuncios por palabras, en algunos medios gratis, como en Segunda Mano-, fueron la espoleta de lanzamiento hacia otras formas de promocionar los producto en Internet.

Ahora se atraviesa una fase de experimentación. Visto lo sucedido en EE.UU. y comparado con Europa, puede decirse que la situación es muy parecida. El concepto que tienen los lectores es que la prensa impresa tiende a desaparecer, pero nada o muy poco se dice cuánto están durando aquellos medios nativos digitales que se crean. Están uno o dos años en el mercado y luego desaparecen. Un reciente estudio del Reuters Institute for the Study of Journalism (RISJ), citado por Cruz señala que: "Las empresas informativas europeas exclusivamente digitales tienen muchos problemas para cubrir sus costes y suelen mantenerse a flote menos de tres años" (Cruz, 2012d). Se analizaron nueve webs de medios digitales nativos y solo dos Mediapart (Francia) y Perlentaucher (Alemania) tenían éxito: uno cobrando por los contenidos y el otro con un modelo de negocio muy diversificado. 
Hay que plantear nuevos retos también en lo digital, pues a la vez que desaparecen cabeceras impresas, muchas digitales también caen. Al final, el camino lo van a marcar dos actores: "Los dos principales retos de los medios nativos digitales son, primero, que el mercado de la información online sigue estando dominado por las versiones digitales de los grandes medios, y segundo, que Google (epígrafe siguiente) y otras grandes empresas controlan el negocio de la publicidad online, haciendo muy difícil que los actores pequeños y medianos puedan generar ingresos significativos" (Cruz, 2012c).

Existe una postura más reciente e intermedia entre las anteriores, de un periódico de EE.UU., cuando se pregunta: “¿Qué tipo de anuncios están desarrollando las empresas? The Wall Street Journal señala que las compañías están probando y encontrando algo de éxito con los anuncios de búsqueda" (Garcimartín, 2013).

A comienzos de febrero de 2013 se dieron a conocer los resultados del periódico The New York Times. Por primera vez en su historia, según AFP, los ingresos procedentes de sus lectores y suscriptores superaron a los obtenidos por la publicidad en 2012. Y esto supuso un aumento en sus beneficios. "Por primera vez en nuestra historia, los ingresos por circulación anual superaron a los de la publicidad. Nuestro modelo de pago continuó mostrando su eficacia por sí mismo", dijo Mark Thomson (Thomson, 2013), el exjefe de la BBC, que pasó a ser presidente y director ejecutivo de The New York Times el pasado noviembre (AFP, 2013). Según esta agencia de información, este periódico está inmerso en un proceso de transición del papel al medio digital. Aún así, consiguió en el cuarto trimestre triplicar sus beneficios (176,9 millones de dólares). De ellos, 164 millones son de la venta de la página de búsqueda de empleo en línea Indeed.com.

Este dato es importante, pues marca las pautas de otros medios impresos y digitales en diarios de todo el mundo. Hay editores que consideran que lo gratis se tiene que acabar; al menos en las informaciones. Thomson, presidente y director ejecutivo, cree firmemente en ese proceso: "La demostrada predisposición de los usuarios de aquí y de todo el mundo a pagar por el periodismo de alta calidad por el que se hizo famoso y es reconocido The New York Times y otras cabeceras de la empresa" (Thomson, 2013).

Hay que lograr atraer nuevos lectores y conocerlos, pues de esta manera la inversión publicitaria crece, dado que los anunciantes tienen claro cuál es su nicho de mercado. "No solo los medios de pago buscan fórmulas que atraigan y fidelicen a su audiencia. Otros completamente gratuitos, como The Guardian, también tratan de hacer crecer su comunidad, y que ésta se sienta parte del periódico. El beneficio, para estos medios, es que los hábitos de sus usuarios se pueden vender a los anunciantes" (Cruz, 2012b).

Mientras se ejecutan esas acciones, otras empresas están ya utilizando el vídeo para aumentar su publicidad. Así, YouTube conecta cuatro mil millones de visitas por día y es el segundo mayor motor de búsqueda en todo el mundo. Entonces, ¿qué negocio le queda al periódico impreso para atraer lectores? ¿Cómo hacer frente a la cultura audiovisual de las nuevas generaciones? ¿Deben buscar nuevas habilidades en la construcción de las noticias para atraer lectores? ¿Será necesario asociarse con sitios de Internet que garantizan con sus visitas millones de lectores? 


\subsection{Google frente a todos y contra todos}

Los medios impresos, y en menor medida los digitales, están atravesando serias dificultades financieras en Estados Unidos. Esto sucede especialmente, cuando los medios importantes, que marcan pautas en el devenir de otros periódicos, no encuentra el camino de los beneficios en su versión impresa y se desacelera en la digital. Es el caso de The New York Times, quien en el tercer trimestre de 2012 anunció un descenso en sus ganancias, pasando de 21 millones en 2011, a 8,1 en 2012. Este descenso se debe sobre todo a la caída de un $10 \%$ de la publicidad en la edición en papel y un $2 \%$ en la Web. Es curioso ese descenso de la Web, ya que se mantenía al alza en anteriores estudios.

Por su parte, la Asociación de Periódicos Americanos (NAA, por sus siglas en inglés) ofrece una visión más completa, ya que su estudio toma como referente una fecha más amplia. Así, los ingresos publicitarios de la prensa en EE.UU. han caído un $55,2 \%$ desde el año 2006, a pesar de que han aumentado sus ingresos a través de los medios digitales un $18,8 \%$, pero no es suficiente para presentar cuentas saneadas. En concreto, los ingresos publicitarios en 2006 fueron de 11.791 millones de dólares, cifra que ha descendido hasta los 5.272 de 2012 . Y lo que es peor, la inversión de medios digitales no compensa esta caída (NAA, 2013).

Es tiempo de evaluar todos los fenómenos que se están produciendo. Los medios, tanto impresos como digitales, deben conocer mejor las necesidades de sus lectores, para poder atraer nuevamente importantes cantidades. El número es vital para la publicidad. En el caso de los anuncios, también en la información, ese cambio social es especialmente significativo en Estados Unidos. Un reciente estudio de Ogilvy: The Cross Cultural Report señala que en un periodo de 30 años (de 1980 a 2010), la población blanca descendió un 15,1 \% en EE.UU. Por el contrario, los hispanos, asiáticos y afroamericanos aumentaron un 7,4, un 3,3 y un $0,9 \%$, respectivamente. "The American population has changed profoundly and will continue to evolve in the years ahead. In fact, as these demographic changes grow in magnitude, the whole notion of what constitutes a minority must surely change" (Bowman, 2012).

Eso significa que las estrategias publicitarias tienen que variar su rumbo. En consecuencia, el tipo de información que deben elaborar los medios impresos y digitales, también debe cambiar. En el tercer trimestre del 2012, según Europa Press, los ingresos publicitarios de la prensa impresa cayeron un $6,4 \%$ con respecto al mismo periodo de 2011. En el caso de los digitales aumentó un 3,6\%.

Mientras la mayoría de los periódicos experimenta todo tipo de fórmulas, especialmente el cobro por suscripción, hay una compañía que parece tener respuestas para todos y para todo: Google. Es el lugar preferido de los usuarios de Internet. Es raro que no lo visiten al menos una vez al día. Eso supone una audiencia millonaria. A la publicidad le interesan sitios como ése. Antes, los medios de comunicación eran capaces de sumar audiencias millonarias frente a la televisión o un medio impreso, pero ninguno de ellos, ni siquiera todos juntos, pueden hacer sombra al gigante Google. De hecho, a finales del 2012, los anunciantes habían invertido más en Google (8.567 millones en todo el mundo) que en todos los medios impresos de EE.UU. (8.260 millones de euros), según señala el periodista Juan Manuel García Campos: “En 
el primer semestre de 2012, el buscador ha facturado más que todas las publicaciones de EE.UU. juntas, y cuatro veces más que todas las españolas" (García Campos, 2012).

En la misma información, se hace una comparativa con España: tomando como referencia los datos de InfoAdex, Google habría ingresado cuatro veces más en publicidad, que todos los periódicos impresos.

El siguiente gráfico resume este avance considerable de Google:

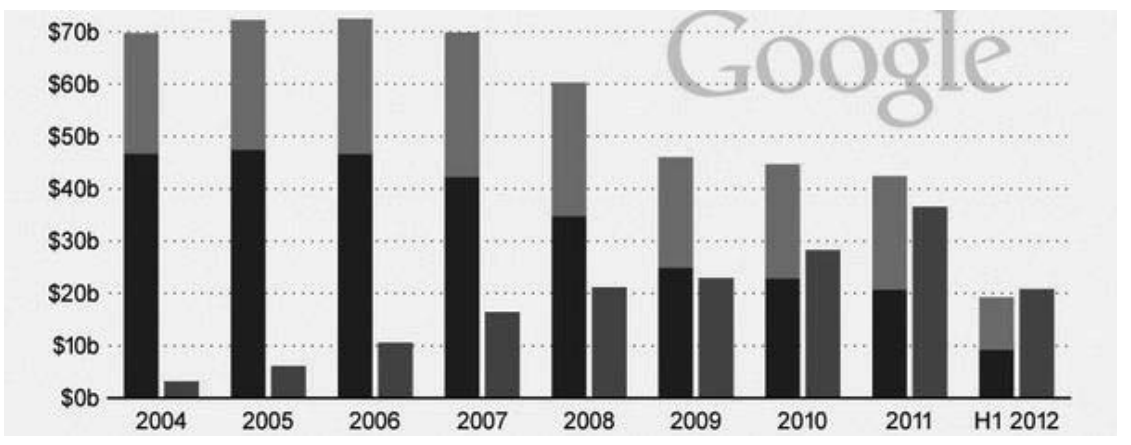

Evolución de la inversión en Google frente a los periódicos impresos en EE.UU.

Fuente: Statisca

El todopoderoso Google tuvo un revés a comienzos del 2013, cuando tuvo que aceptar un acuerdo en Francia, que cambiará la forma en que se distribuye la información y reportará nuevos ingresos a los periódicos franceses. El acuerdo entre Google y los editores de prensa, que venían reclamando como en otros países parte de los beneficios publicitarios que obtenía dicha compañía, se cifra en 60 millones de euros, para un fondo que ayude a los medios en su transición hacia lo digital. Se anunció en su presentación, que cuando se acaben esos 60 millones, se volverá a negociar un nuevo acuerdo.

Previamente a estas negociaciones, el gobierno francés había amenazado a Google con legislar algún tipo de impuesto. Por su parte, la compañía norteamericana amenazó con dejar de redireccionar las informaciones, aunque al final no lo hizo. Los ingresos que Google obtiene en Francia rondan los 1.000 millones de euros en publicidad, lo que significa que una parte se debe a los contenidos informativos que generan los periódicos franceses, que Google rastrea para hacerlos suyos.

Este cambio significativo es posible que se produzca en otros países; al menos, en aquellos en los que el gigante norteamericano esté interesado. No aporta cantidades para salvar la mala situación que atraviesan los medios impresos y además obliga a invertir en temas digitales e innovación, con lo que cierra el paso a los medios impresos donde Google no puede competir.

No es sólo Google el que ha logrado importantes sumas de publicidad frente a los medios impresos. Otras redes sociales, como Facebook y Twitter, también están consiguiendo importantes inversiones que van en detrimento de la realizada en periódicos. Y el negocio de la publicidad se extiende a los móviles y las tabletas. Aquí tienen mucho que decir los periódicos, ya que todos han adaptado su versión digital a estos nuevos soportes. Pero una vez más llegan tarde. Google consiguió en el tercer cuatri- 
mestre de 2012, 150 millones de dólares, es decir, un 14\% de sus ingresos totales en publicidad. Según un estudio de e-Marketer, el gigante norteamericano: "Se embolsa un $56 \%$ de todo el dinero que producen los anuncios para móviles y un $96 \%$ de las búsquedas realizadas con esos dispositivos. La empresa cree que posiblemente ganará 8.000 millones de dólares el año que viene gracias a las ventas a través del móvil" (Cain Miller, 2012).

Los móviles y las tablets son otra opción para conseguir publicidad en los medios digitales. Disponen de información de calidad, de fotografías, vídeos, de contenidos informativos de actualidad: con esos servicios deberían lograr publicidad antes de que compañías ajenas a los medios de comunicación (Google, Facebook, Twitter) se queden con toda la tarta publicitaria.

Pese a todo ello, coincidimos con la advertencia realizada por Armentia: "Sin embargo, hasta ahora la web se ha mostrado especialmente adecuada para las noticias breves (fenómenos como el de Twitter y sus famosos 140 caracteres redundan en este aspecto), pero no ha habido un desarrollo paralelo por parte de géneros específicamente interpretativos. De ahí el peligro de los cibermedios en caer en una excesiva superficialidad a la hora de abordar los temas" (Armentia, 2011).

\subsection{España, un incierto futuro de los medios impresos}

El año 2012 se presentó como un punto de inflexión. Los anunciantes españoles invirtieron más, por primera vez en medios digitales que en impresos. Suponen ya, según datos del IAB Spain, el 18,3\% de la inversión publicitaria, frente al 15,6\% de la inversión en medios impresos: "Por primera vez la inversión en medios digitales (18'3\%) supera a la de medios impresos (15'6\%)" (IAB Spain, 2012)

La televisión, por su parte, sigue en alza con un $43 \%$. Pero, ni siquiera lo digital suma en estos tiempos de crisis, ya que la inversión cayó un $1,1 \%$ en el primer semestre del 2012, lo que supone cinco millones menos que en 2011. De todas estas cifras, quien se lleva la peor parte son los periódicos, pues la publicidad en Internet se desploma y cae un $13 \%$, con lo que la inversión baja hasta el $44 \%$, lo que supone 173,7 millones. De esta cifra, los periódicos reciben la mayor parte, pero cada vez menos.

Otros organismos ofrecen cifras parecidas al estudio de IAB Spain. Así, los datos del Observatorio de la Prensa Diaria, elaborado por Deloitte para la AEDE, hablan de desplome: "El Observatorio de la Prensa Diaria, informe elaborado por la Asociación Española de la Prensa Diaria (AEDE) y la consultora Deloitte, concluye que el 2010 ha sido un año catastrófico para los periódicos españoles: caída generalizada de la difusión, de la venta de ejemplares, de las suscripciones y de contratación publicitaria" (Navas, 2011). Hasta el mes de noviembre de 2012, es decir, casi todo el año, dicho Observatorio señala una caída en la inversión publicitaria de un $18,9 \%$, siendo más significativa en El País (-255\%), ABC y El Mundo (-19\%), La Razón (-16,85), El Periódico de Cataluña (-13,5\%), La Voz de Galicia (-18,6\%), Cinco días (-22\%) y Expansión (-23\%). Estas cifras son parecidas a las que maneja el experto en inversiones en Publicidad, InfoAdex que concreta un descenso de un $-21 \%$ en la publicidad de los diarios. 
Otro empresa que estudia las cifras de inversión publicitaria en los medios es Zenith Vigía, formado por directivos de medios de comunicación. Sus cifras para el 2012 fueron una caída de un 14,6\%: "La inversión publicitaria en medios podría caer este año un 11,2\%" (Zenit, 2013). En concreto, las cifras que aporta este estudio son: Diarios de pago, una caída del 19,1\%; diarios gratuitos, un $10,1 \%$; revistas, un $15,3 \%$; y suplementos, un $15,5 \%$. Lo peor es que las previsiones no son buenas, ya que para el 2013 consideran que la caída será de un 9,1\%. Además, hay una coincidencia entre anunciantes y medios de comunicación, en que el mercado no mejorará ni se recuperará hasta 2014, en concreto hacia el mes de abril de ese año.

Por último, otra de las empresas que hace un seguimiento importante en la inversión publicitaria en España, InfoAdex, presenta también cifras negativas para los medios: "La inversión real estimada en Medios Convencionales alcanzó los 4.630,0 millones de euros durante el año 2012, cifra que representa un decrecimiento del $15,8 \%$ sobre la registrada en el año anterior. Vuelve así a producirse en 2012 una contracción del mercado, tal y como ya ocurrió en los años 2008, 2009 y 2011" (InfoAdex, 2013). En concreto, da como última cifra aceptable 2010, donde la caída con respecto a los años anteriores se frenaba. Ese año la inversión en prensa subía hasta los 1.124,4 millones de facturación, cayendo hasta los 967 en 2011. Para 2012, se reducía considerablemente la inversión hasta el mes de junio, quedando en 370 millones. En sus previsiones para el año 2013, tampoco hay una mejoría, ya que se estima en torno a unos 700 millones la inversión en prensa.

Si la mayoría de estudios, asociaciones y organismos especializados en inversión publicitaria coinciden, sólo queda una posible vía para mejorar los ingresos en las empresas editoras de medios: mejorar los contenidos del formato digital, para que de esta manera se aumenten los ingresos. Todo ello va en detrimento de la versión impresa, que seguirá perdiendo, tal y como señalan todos los análisis efectuados. Es más, hasta incluso algunos editores consideran que el ciclo de inversiones en medios impresos se está cerrando.

Así lo expuso, a finales de 2012, el presidente del Grupo Prisa, Juan Luis Cebrián, durante la 68 Asamblea General de la Sociedad Interamericana de Prensa (SIP): "Hay una amenaza real, debido a la estructura económica del sistema, a la calidad periodística si no encontramos un modelo o varios modelos que permitan monetizar toda nuestra actividad en la red" (El Economista, 2012). Algunos expertos asistentes al acto puntualizaron las palabras del presidente de Prisa, al indicar que: "El mecanismo de financiación de la prensa en papel a través de la publicidad está agotado desde la irrupción de Internet y no se vislumbra un único modelo de éxito para el futuro"(El Economista, 2012).

Por tanto, en lo que sí hubo acuerdo fue en que la inversión publicitaria ya no regresará a los periódicos: no al menos como el sustento principal de la inversión. Además, se necesitarán otros modelos para que la prensa impresa pueda continuar. En palabras de Rosental Calmon Alves (El Economista, 2012), en el mismo encuentro y refiriéndose a la inversión publicitaria de los medios impresos: "no va a volver". Esta situación exige un mayor compromiso por parte de los periodistas: mejorar la información, ofrecer más calidad que cantidad y algo distinto y superior al gratuito en la Web. 
Es curioso que en esta transformación nadie calculó el descenso de las inversiones en publicidad, sabiendo que eran el principal factor de ingresos en los medios. La mayoría de los editores de prensa impresa se pasaron a lo digital sin más, creyendo que el futuro de la empresa pasaba por ofrecer un periódico online. Pero casi nadie advirtió que la publicidad en Internet no acudía sumisa y dócil, como antes.

A estas alturas, una década larga después de la implantación de los periódicos digitales, se cuentan con los dedos de una mano los editores que pueden aportar beneficios en la versión digital de su medio. Dos datos del presidente de Prisa avalan esta situación: "Ningún periódico que haya realizado una migración del papel a las versiones digitales ha tenido éxito en términos de rentabilidad económica" y "Por cada dólar neto de publicidad que se genera en las redes se destruyen 10 en papel" (El Economista, 2012).

No resulta, pues, exagerado afirmar que el negocio de los periódicos impresos está muriendo y que tampoco los medios digitales tendrán garantizada su existencia; al menos, mientras Google y algunas redes sociales sean capaces de concentrar más usuarios que los propios medios digitales. Queda claro que hay una transición menor de diez años en la que ambos formatos tendrán que buscar su nicho de mercado. Es necesario adecuar su modelo de negocio a las circunstancias actuales del mercado.

La publicidad, para ser efectiva, se cambia a otros lugares de Internet que, como Google o las Redes Sociales, son sitios con millones de usuarios dispuestos a comprar sus productos. Es, por tanto, urgente que los periódicos impresos trabajen en dos frentes: a) Mejorar la calidad de la información, con el uso de numerosas fuentes de información y apoyos documentales, aportando datos, hechos y opiniones que den una información inaccesible en Internet ni en las Redes Sociales; b) Crear publicidad muy segmentada, dirigida a lectores dispuestos a pagar por una información diferenciada y exclusiva.

Ese cambio puede resultar traumático para las redacciones, más aún de lo que ha venido siendo en los tres últimos años. Juan Luis Cebrián (El Economista, 2012) anunció recientemente que se perderían en el transcurso de los próximos tres años, cerca de 7.000 periodistas, con lo que coloca a esta profesión en España al borde del abismo. De estas cenizas tendrá que resurgir el nuevo periodismo español: un nuevo modelo de información impresa sin publicidad, o con muy poca publicidad, que necesitará el pago de contenidos por parte de los lectores.

\section{Conclusiones}

Durante los próximos diez años se asistirá a un cambio de paradigma en los medios impresos. Desde la estructura de las redacciones, con intensos recortes que aún no han finalizado, pasando por la elaboración de nuevas formas de comunicar y contando con menos recursos financieros ante el descenso de la inversión publicitaria. Este ajuste servirá para ir manteniendo cabeceras tradicionales, que serán las que sobrevivan, si se adaptan a los cambios y, junto a ellas, periódicos más locales, con lectores interesados en informaciones de su comunidad.

Nadie es capaz de augurar cuáles y cuántas serán las vías de financiación de los medios impresos. Pero el cambio se ha de producir, si no quieren desaparecer: con el nú- 
mero de suscriptores y el pago por ejemplar, ya hace años que los medios impresos deberían haber muerto. Ahora, en pleno descenso de la inversión publicitaria, los periódicos y las revistas han tenido que regenerar su negocio. Primero, adelgazando las plantillas, y luego buscando alternativas para atraer lectores, que por otra parte, ya se han desplazado a lo digital. Se trata, en definitiva, de buscar modelos que sean económicamente sustentables para los medios impresos.

Es difícil que con un modelo caduco del siglo XX, se consigan hoy los pingües beneficios ansiados de aquellas épocas. Los periódicos impresos españoles tardaron en adaptarse al nuevo sistema digital y ahora están pagando parte de sus consecuencias. Estamos asistiendo al fracaso de la industria de los medios impresos, por no saber adaptarse a las necesidades de un nuevo mercado.

Las noticias que en el siglo XX tenían un valor de al menos 24 horas, en los tiempos actuales se cuentan por segundos. Tampoco se ha mejorado a la hora de hacer el producto digital, pensando que sólo sería un apoyo más y que su verdadero negocio sería, como hasta entonces, el medio impreso. Los editores de prensa no supieron calcular el riesgo que suponía la llegada de Internet, y menos aún de las redes sociales. Esos cambios tan profundos deberían haberse contemplado mucho antes y deberían haber sido medidos y calibrados de otra forma.

Otro de los aspectos que no se acertó a cuidar fue el tema de las audiencias. Cautivas de los medios impresos, que se acercaban cada día al kiosco a comprar, en Internet tienen ahora plena libertad para conseguir ellos mismos las informaciones: en muchos casos de manera gratuita; en otros pagando una parte, o presentando modelos Freemium en los que el lector paga por lo que tiene un valor para él.

El camino más inmediato es buscar un modelo de negocio sustentable, que ofrezca un equilibrio entre lo que cuesta hacer la información y lo que aporta la publicidad como inversión. A los editores les queda la esperanza de que el modelo digital, el implantado por The New York Times y otros medios anglosajones, reporte ya más beneficios que la publicidad y, en consecuencia, sirvan para sustentar el medio impreso. Es cierto que el negocio de los medios se encamina a lo digital y que los recursos que pueden generar los periódicos impresos serán muy limitados, pero la conclusión más importante es que así como en el digital funciona el sistema de pago por suscripción, en la versión impresa debería volver a atraer lectores de pago y suscriptores, a cambio de ofrecer información y opinión de calidad.

\section{Referencias bibliográficas}

ARMENTIA VIZUETE, José Ignacio (2011): "La difícil supervivencia de los diarios ante la agonía del soporte papel", en Ámbitos, no 20, p. 21, pp. 11-27. http://grupo .us.es/grehcco/ambitos20/01armentia.pdf [fecha de consulta 12/04/2013]

BOWMAN, Jeffrey (2012): "The Cross-Cultural Report”, p. 7, 37 págs., en: http://www.ogilvy.com/On-Our-Minds/Articles/2012_Oct_CrossCultural-Report.aspx [fecha de consulta 21/10/2013]

BREINER, James (2012): "How to tailor news for 4 different platforms? 'Responsive design"', en News Entrepreneurs. Business models for new digital media, 25 
October, en: http://newsentrepreneurs.blogspot.com.es/2012/10/how-to-tailornews-for-4-different.html_[fecha de consulta 18/10/2013]

BRENNER, Joanna (2013): “Pew Internet: Mobile”, en Pew Internet \& American Life Project, 18 September, en: http://pewinternet.org/Commentary/2012/February /Pew-Internet-Mobile.aspx_[fecha de consulta 18/10/2013]

CABRERA GONZÁLEZ, María Ángeles, (coord., 2010): Evolución tecnológica y cibermedios. Málaga, Ed. Comunicación Social, Col. Periodística nº 34, 188 págs, p. 149.

CAIN MILLER, Claire (2013): "La publicidad apunta a móviles y tabletas", en $E l$ Pais, 15-11-2012, en: http://tecnologia.elpais.com/tecnologia/2012/11/15/actualidad/1352992597_587492.html [fecha de consulta 12/04/2013]

CARRETERO, Rodrigo (2012): "La barra libre en Internet puede estar terminando", en MediaTics.com, 8 de noviembre, http://www.media-tics.com/noticia/2524/Medios-de-Comunicacion/La-barra-libre-en-Internet-puede-estar-terminando.html [fecha de consulta 23/10/2013]

CEBRIÁN, Juan Luis (2012): SIP (68 Asamblea General de la Sociedad Interamericana de Prensa), "En España 7.000 periodistas perderán su empleo en tres años" en El Economista, 13 de octubre, en: http://ecoteuve.eleconomista.es/empresas-finanzas/noticias/4320525/10/12/Cebrian-En-Espana-7000-periodistas-perderan-suempleo-en-tres-anos.html [fecha de consulta 14/10/2013]

CRUZ, Manuela (2012a): "El 60\% de los editores coincide: los periódicos serán solo digitales en 2020", en MediaTics.com, 03-04-2012, http://www.media-tics.com/noticia/1618/Medios-de-Comunicacion/El-60-de-los-editores-coincide:-los-periodicos-seran-solo-digitales-en-2020.html [fecha de consulta 23/10/2013]

CRUZ, Manuela (2012b): "Para los periódicos digitales la clave es saber quién les visita, no cuántos son", en MediaTics.com, 3 de abril, en: http://www.mediatics.com/noticia/1585/Medios-de-Comunicacion/Para-los-periodicos-digitales-la-cl ave-es-saber-quien-les-visita-no-cuantos-son.html [fecha de consulta 12/04/2013]

CRUZ, Manuela (2012c): "Los periódicos nativos digitales sobreviven menos de tres años", en MediaTics.com, 3 de mayo, en: http://www.media-tics.com/noticia/1754/Medios-de-Comunicacion/Los-periodicos-nativos-digitales-sobrevivenmenos-de-tres-anos.html [fecha de consulta 21/10/2013]

CRUZ, Manuela (2012d): "Medios de Comunicación. Según un estudio, tienen dificultades para cubrir costes Los periódicos nativos digitales sobreviven menos de tres años", en MediaTics.com, 3 de mayo, http://www.media-tics.com/noticia/1754/ [fecha de consulta 23/10/2013]

DAWSON, Ross (2006): “Future of Media", en 2006 - 2013 Future Exploration Network, http://futureexploration.net/future-of-media_[fecha de consulta 18/10/2013]

DIRIGENTES DIGITAL (2012): "El futuro de las noticias económicas es móvil”, 19 de noviembre, en: www.dirigentesdigital.com/articulo/estrategias/209743/futuro/no [fecha de consulta 14/10/2013] 
DURRANI, Arif (2012): “Rob Grimshaw talks mobile and the FT's biggest challenge yet", en MediaWeek, 7 November: http://www.mediaweek.co.uk/news/1158494/ [fecha de consulta 23/10/2013]

EDITOR\&PUBLISHER (2012): "Survey Finds $60 \%$ of Publishers Agree that Print Publishing's Time is Limited posted: 3/9/2012", en: http://www.editorandpublisher.com/Business/Article/Survey-Finds-60 - of-Publishers-Agree-that-Print-Publishing-s-Time-is-Limited\#sthash.IEc3pPgG.dpuf [fecha de consulta 14/10/2013] ESTUDIO INFOADEX DE LA INVERSIÓN PUBLICITARIA EN ESPAÑA (2013): http://www.infoadex.es/Nota_Prensa_Estudio_InfoAdex_Inversion_Publicitaria_Espana_2013.pdf_[fecha de consulta 14/10/2013]

GARCÍA CAMPOS, Juan Manuel (2012): "Google ya supera a toda la prensa de EE.UU. en ingresos publicitarios", en La Vanguardia.com, Marketing y Publicidad, Barcelona 15 de noviembre, en: http://www.lavanguardia.com/economia/marketing-publicidad/20121115/54354519644/google-ya-supera-a-toda-la-prensa-de-estados-unidos-en-ingresos-publicitarios.html_[fecha de consulta 21/10/2013]

GARCIMARTÍN, Miriam (2013): "Móviles y tabletas. El futuro de la publicidad es móvil... e incierto", en MediaTics.com, 28 de febrero, en: http://www.mediatics.com/noticia/2868/Moviles-y-tabletas/El-futuro-de-la-publicidad-es-movile-incierto.html [fecha de consulta 23/10/2013]

HARRIS, Jim (2012): "What's Really Killing Print Journalism", en Huffington Post, Canadá, 24 October, en: http://www.huffingtonpost.ca/users/becomeFan.php? of=hp_blogger_Jim\%20Harris [fecha de consulta 23/10/2012]

HURST, Marcus (2012): "El periodista que recaudó 3.600 euros en una labor de crowdfunding inverso", en Yorukobu, 22 de octubre, en: http://www.yorokobu.es /el-periodista-que-recaudo-3-600-euros-en-una-labor-de-crowdfunding-inverso/ [fecha de consulta 14/10/2013]

IAB SPAIN (2012): "Por primera vez la inversión publicación en medios digitales $\left(18^{\prime} 3 \%\right)$ supera a la de medios impresos $\left(15^{\prime} 6 \%\right)$ ", 22 de octubre, en: http://www.iabspain.net/noticias/por-primera-vez-la-inversion-publicitaria-en-medios-digitales-183-supera-a-la-de-medios-impresos-156/_[fecha de consulta 14/10/2013]

KIRKLAND, Sam (2012): Highlights from Poynter's Eyetrack Tablet Conference, 19 October, en: http://www.poynter.org/how-tos/newsgathering-storytelling/visualvoice/192252/follow-poynters-eyetrack-conference-online/ [fecha de consulta 18/10/2013]

LE MONDE (2012): "Le Monde" se transforme", 19 novembre, en: http://www.lemonde.fr/actualite-medias/article/2012/11/19/le-monde-nouvelleetape_1792245_3236.html [fecha de consulta 14/10/2013]

MATTER (2012): en Journalism.co.uk, 4 July, en: http://www.journalism.co.uk /news/digital-long-form-journalism-project-matter-aiming-for-septemberlaunch/s2/a549767/ [fecha de consulta 14/10/2013] 
NAVAS GARCÍA, Alejandro (2011): "La sociedad de convergencia", en ALCUDIA, Mario et al. (coords): Competidores y aliados: Medios en convergencia, los nuevos retos en comunicación. Madrid, CEU Ediciones, p.24. Cfr. www.aede.es

NEWS\&TECH (2012): "20 trends for 2020", 30 August, en: http://www.newsandtech.com/news/article_a457861e-f2de-11e1-b615-0019bb2963f4.html_[fecha de consulta 18/10/2013]

NEWSPAPER ASSOCIATION OF AMERICA, ADVANCING NEWSPAPER MEDIA (2012): "The American Newspaper Media Industry Revenue Profile 2012", en: http://www.naa.org/Trends-and-numbers/newspaper-Revenue/newspaper-mediaIndustry-Revenue-Profile-2012.aspx [fecha de consulta 21/10/2013]

RUIZ, Ana (2012): "La suscripción digital reportó a The New York Times 91 millones de dólares en 2012", en MediaTics.com, 28 de febrero, en: http://www.mediatics.com/noticia/2787/ [fecha de consulta 23/10/2012]

SAMPLE (2012): "State of Search Marketing Report 2012, in association with SEMPO", New York-London, 2012, p. 6, http://www.slideshare.net/joaocaetano/sample-sempo-state-of-search-marketing-report-2012 [fecha de consulta 23/10/2012]

THE TELEGRAPH (2012): “Teenager launches Apple iPhone app after raising \$1m from investors", 1 September, en: http://www.telegraph.co.uk/finance/newsbysector/mediatechnologyandtelecoms/digital-media/9649209/Teenager-launchesApple-iPhone-app-after-raising-1m-from-investors.html [fecha de consulta 14/10/2013]

THOMSON, Mark, (2013): “The New York Times, 162 años de evolución y reinvención", Yahoo Noticias, 18 September, en: http://es-us.noticias.yahoo.com/thenew-york-times- $162-\mathrm{a} \% \mathrm{C} 3 \% \mathrm{~B} 1$ os-evoluci\%C3\%B3n-reinvenci\%C3\%B3n-17010 4898 - finance.html [fecha de consulta 23/10/2013]

WALES, Jimmy (2013): "La prensa online será sostenible cobrando", en El Mundo, 27 de abril, Mercados, p. 12, por César Urrutia [fecha de consulta 23/9/2013]

ZENITH VIGÍA (2012): "La inversión publicitaria en medios podría caer este año un 11,2\%" "Inversión en Medios: la salida de la crisis más cerca", 7 de octubre, en: http://www.blogginzenith.zenithmedia.es/tag/zenith-vigia/ [fecha de consulta $14 / 10 / 2013]$ 